\title{
Coeficiente de erodibilidade em sulcos e entressulcos de Argissolos coesos estimado pela cor do solo
}

\author{
Jussara Silva Dantas(1), Marcílio Vieira Martins Filho(2), José Marques Júnior ${ }^{(2)}$, \\ José Maria do Amaral Resende( ${ }^{(1)}$, Daniel De Bortoli Teixeira(2), Ronny Sobreira Barbosa ${ }^{(2)}$ \\ e Diego Silva Siqueira(2)
}

\begin{abstract}
(1)Universidade Federal do Maranhão, Centro de Ciências Agrárias e Ambientais, BR-222, Km 04, s/no, Boa Vista, CEP $65500-000$ Chapadinha, MA, Brasil. E-mail: jussarasd@yahoo.com.br, zemariaresende@bol.com.br (2)Universidade Estadual Paulista, Faculdade de Ciências Agrárias e Veterinárias, Departamento de Solos e Adubos, Via de Acesso Prof. Paulo Donato Castellane, s/no, CEP 14884-900 Jaboticabal, SP, Brasil. E-mail: mfilho@fcav.unesp.br, marques@fcav.unesp.br, daniel.dbt@hotmail.com, rsbagronomo@gmail.com, diego_silvasiqueira@yahoo.com.br
\end{abstract}

Resumo - O objetivo deste trabalho foi estimar o coeficiente de erodibilidade em sulcos e entressulcos, por meio de parâmetros da cor do solo. Foram selecionadas três áreas cultivadas com soja, em Argissolos Amarelos coesos do Leste Maranhense. Em cada área, foram retiradas 121 amostras na profundidade de 0,00-0,20 m. Foram determinados os valores de espectroscopia de reflectância difusa na faixa do visível, para obtenção dos valores de matiz, valor e croma. A partir destes constituintes da cor, foi calculado o índice de avermelhamento para cada amostra. Foram desenvolvidos modelos para avaliar o efeito de cada componente da cor sobre a erodibilidade em sulcos e entressulcos. Esses componentes apresentaram $\mathrm{R}^{2}$ que variou de 0,37 a 0,70 . Os modelos gerados obtiveram $\mathrm{R}^{2}$ de 0,70 e 0,73 , para entressulcos e sulcos, respectivamente. A cor do solo determinada por espectroscopia de reflectância difusa pode ser utilizada como método alternativo para estimar o coeficiente de erodibilidade de Argissolos Amarelos coesos.

Termos para indexação: cerrado, erosão do solo, espectroscopia de reflectância difusa, Formação Barreiras, funções de pedotransferência, pedometria.

\section{Coefficient of rill and interrill erodibility of cohesive Ultisols estimated by soil color}

\begin{abstract}
The objective of this work was to estimate the rill and interrill erodibility coefficient through soil color parameters. Three areas were selected, which were cultivated with soybean in cohesive Ultisols of the eastern region of the state of Maranhão, Brazil. In each area, 121 samples were collected at a depth of $0.00-0.20 \mathrm{~m}$. The values of diffuse reflectance spectroscopy in the visible range were determined, in order to obtain the values of hue, value, and chroma. From these color constituents, the redness index was calculated for each sample. Models were developed to evaluate the effect of each color component on rill and interril erodibility. These components showed $\mathrm{R}^{2}$ ranging from 0.37 to 0.70 . The generated models obtained $\mathrm{R}^{2}$ of 0.70 and 0.73 for interill and rill, respectively. Soil color determined by diffuse reflectance spectroscopy can be used as an alternative method to estimate the erodibility coefficient of cohesive Ultisols.
\end{abstract}

Index terms: cerrado, soil erosion, diffuse reflectance spectroscopy, Formação Barreiras, pedotransfer functions, pedometry.

\section{Introdução}

O desenvolvimento da agricultura tem aumentado a demanda por informações rápidas e detalhadas sobre os atributos e, principalmente, sobre o potencial de erosão dos solos (Rockstrom et al., 2009; Quinton et al., 2010), com o intuito de balizar a produção vegetal, minimizar os impactos ambientais e, consequentemente, diminuir a pressão sobre o uso e a ocupação do solo.
O conhecimento da taxa de erosão do solo é importante, tanto para a compreensão da evolução do relevo quanto para avaliar o impacto da atividade humana sobre esses solos (Parsons et al., 2010). Para modelar o processo de erosão hídrica do solo, destacam-se os modelos da equação universal de perda de solo (USLE) (Wischmeier \& Smith, 1978) e do projeto de predição de erosão hídrica (WEPP) (Lane \& Nearing, 1989). O modelo USLE é teórico/ 
empírico e o WEPP é determinístico; ambos utilizam parâmetros relacionados ao processo de erosão do solo, entre eles a erodibilidade (Sheridan et al., 2000).

No modelo WEPP, são utilizados dois coeficientes de erodibilidade: entressulcos e sulcos (Lane \& Nearing, 1989), em condições de estado de equilíbrio dinâmico quanto à chuva e à enxurrada. Esses coeficientes podem ser mensurados diretamente por experimentos de chuva artificial ou natural, ou estimados a partir de propriedades do solo pelo uso de equações provenientes de procedimentos de regressão. Essas equações utilizam atributos correlacionados aos coeficientes de erodibilidade, como granulometria, teor de matéria orgânica e composição química, entre outros (Flanagan \& Livingston, 1995; Sheridan et al., 2000).

A erosão em sulcos e entressulcos tem sido utilizada para avaliar diferentes tipos de preparo e manejo do solo (Cassol \& Lima, 2003) e para compreender a relação de chuvas intensas com a erosão hídrica do solo (Santos et al., 2010). A separação do processo de erosão em sulcos e entressulcos pode ajudar a identificar fontes potenciais de sedimentos, o que é essencial para a modelagem da distribuição de insumos agrícolas nas áreas de produção, especialmente daqueles que são fortemente adsorvidos pelas partículas do solo (Martins Filho et al., 2009; Andrade et al., 2011). No entanto, a quantificação da erodibilidade do solo ainda é bastante demorada e muito onerosa, uma vez que ela depende da determinação de outras propriedades do solo. Assim, alguns pesquisadores têm proposto o desenvolvimento de funções de pedotransferência para estimar propriedades do solo complexas ou de alto custo de determinação, por meio de propriedades mais simples ou de menor custo (McBratney et al., 2002; Minasny \& Hartemink, 2011). Entre essas funções, ressaltase a determinação de atributos do solo por meio da suscetibilidade magnética (Siqueira et al., 2010; Peluco et al., 2013; Santos et al., 2013) e da espectroscopia de reflectância difusa (Barrón et al., 2000; Torrent \& Barrón, 2008; Viscarra Rossel \& Webster, 2011).

A cor do solo é um atributo diagnóstico que está relacionado à presença de óxidos de ferro e de outras importantes propriedades do solo (Barrón et al., 2000). Porém, a praticidade da determinação da cor pela carta de Munsell não compensa a sua subjetividade e a falta de precisão para informações quantitativas.
Nesse sentido, as informações fornecidas por meio da espectroscopia de reflectância difusa em amostras, no campo ou no laboratório, podem ser utilizadas para refinar o sistema de cor de Munsell, o que gera nova ferramenta de gestão ainda mais prática e precisa (Barrón et al., 2000; Torrent \& Barrón, 2008). Esse refinamento atribui valores numéricos precisos para cada nova cor gerada, que pode ser associada aos valores dos atributos do solo obtidos nas análises convencionais. Viscarra Rossel \& Webster (2011) conciliaram a quantificação de atributos da cor com uso de análises geoestatísticas, ao relacionar os resultados a diferentes compartimentos climáticos e da paisagem.

A cor do solo determinada por espectroscopia de reflectância difusa tem sido utilizada para elaboração de funções de pedotransferência, em diferentes níveis de detalhe e para diversos fins. Também pode ser utilizada como técnica alternativa de gestão, de maneira mais prática e precisa do que o sistema de Munsell, o que permite que parâmetros da cor do solo (matiz, croma e valor) sejam utilizados como método alternativo à fórmula de Flanagan \& Livingston (1995), na predição da erodibilidade em entressulcos e sulcos. Segundo Cohen et al. (2007), há evidências de que, por meio de processos analíticos de reflectância espectral do solo, vários atributos podem ser estimados com grande acurácia, principalmente aqueles de difícil mensuração. Dessa forma, essa técnica surge como alternativa para viabilizar o mapeamento de grandes áreas, uma vez que o estudo espectral não apresenta restrições e dificuldades na obtenção dos dados.

O objetivo deste trabalho foi estimar o coeficiente de erodibilidade em sulcos e entressulcos, por meio de parâmetros da cor do solo.

\section{Material e Métodos}

As áreas selecionadas para o estudo localizam-se no Município de Brejo, a leste do Estado do Maranhão $\left(03^{\circ} 36^{\prime} \mathrm{S}, 42^{\circ} 52^{\prime} \mathrm{W}\right.$, a $100 \mathrm{~m}$ de altitude média). O clima da região, conforme a classificação de Köppen, é do tipo Aw, tropical quente e úmido, com precipitação média de $1.835 \mathrm{~mm}$ e com chuvas concentradas no período de dezembro a maio. A vegetação natural é constituída por cerrado ou pela transição cerrado/ floresta subcaducifólia, e a área é atualmente utilizada para o cultivo de soja (Glycine max L.) (Atlas do Maranhão, 2002). O material geológico na área estudada está relacionado aos sedimentos da Formação 
Barreiras e é caracterizado pelo ambiente de formação fluvial do período Terciário (Jacomine et al., 1986).

Foram selecionadas três áreas experimentais (Figura 1). De acordo com o modelo digital de elevação, as áreas I e II situam-se em pedoforma côncava, enquanto a área III se situa em pedoforma convexa. Para fins de complementação, foram abertas trincheiras em cada uma das três áreas de vegetação nativa, e os solos foram classificados como Argissolo Amarelo distrocoeso típico (Santos et al., 2006). Os atributos físicos e químicos dos solos amostrados nos três perfis das áreas experimentais foram caracterizados (Tabela 1).

Nas áreas cultivadas com soja, foram delimitadas três malhas de amostragem quadradas contendo

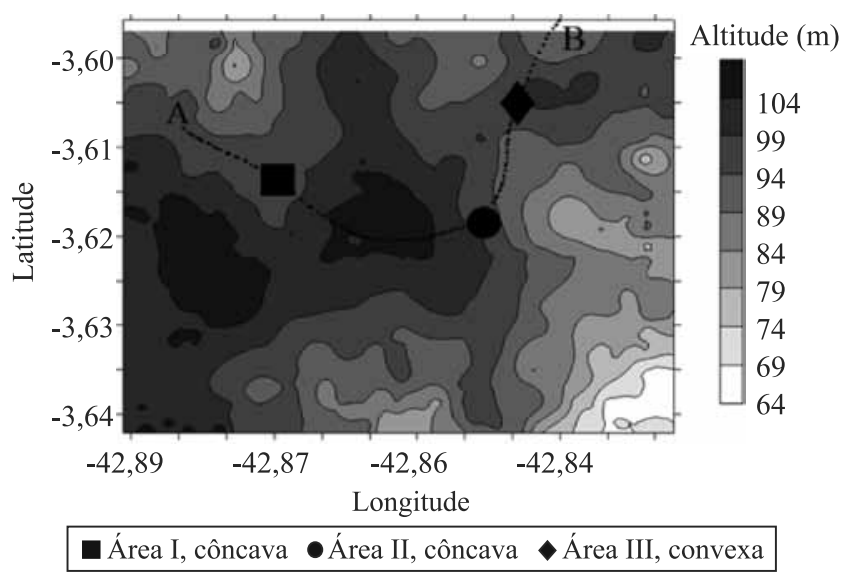

Figura 1. Localização das três áreas do estudo e perfil altimétrico no corte AB.
121 pontos. As malhas das áreas I e II apresentaram espaçamento regular entre amostras de $25 \mathrm{~m}$ e área total de 6,2 ha. A malha localizada na área III apresentou espaçamento regular de $10 \mathrm{~m}$, o que totalizou 2,2 ha. Nas três áreas, foram coletadas 121 amostras nos pontos de cruzamento da malha, na profundidade de $0,00-0,20 \mathrm{~m}$.

A textura do solo foi determinada pelo método da pipeta, com solução de $\mathrm{NaOH} 0,1 \mathrm{~mol} \mathrm{~L}^{-1}$ como dispersante químico e agitação mecânica em aparato de baixa rotação, por 16 horas. A fração argila foi separada por sedimentação; as areias grossa e fina, por tamisação; e o silte, calculado por diferença, de acordo com Donagema et al. (2011).

Para a caracterização da fertilidade do solo, foram determinados cálcio, magnésio, potássio, sódio e fósforo disponível, os quais foram extraídos pelo método da resina trocadora de íons; a acidez trocável $\left(\mathrm{Al}^{3+}\right)$ e a acidez potencial $(\mathrm{H}+\mathrm{Al})$ foram obtidas conforme Raij et al. (2001). Os teores de carbono orgânico foram determinados por oxidação úmida, e o pH foi determinado na relação 1:2,5 de solo em água (Donagema et al., 2011).

Os valores dos coeficientes de erodibilidade em entressulcos (EE) e sulcos (ES) foram calculados para as 363 amostras correspondentes às três malhas de coleta, por meio das equações apresentadas por Flanagan \& Livingston (1995), para solos com teores de areia maiores ou iguais a $300 \mathrm{~g} \mathrm{~kg}^{-1}$ : $\mathrm{EE}=2728000+192100 \times \mathrm{AMF}$ e $\mathrm{ES}=0,00197+0,00030 \times \mathrm{AMF}+0,03863 \mathrm{e}^{(-1,81 \times \mathrm{MO})}$, em que AMF é a areia muito fina (\%), e MO é o teor de matéria orgânica $(\%)$. O coeficiente de EE

Tabela 1. Atributos físicos e químicos de três perfis de Argissolos pertencentes à área experimental no Leste Maranhense ${ }^{(1)}$.

\begin{tabular}{|c|c|c|c|c|c|c|c|c|c|c|}
\hline Hor. & $\begin{array}{l}\text { Prof. } \\
(\mathrm{cm})\end{array}$ & Cor & ------------- & Silte & ---------- & $\begin{array}{c}\mathrm{DS} \\
\left(\mathrm{g} \mathrm{cm}^{-3}\right)\end{array}$ & $\mathrm{pH}$ & $\begin{array}{c}\mathrm{MO} \\
\left(\mathrm{g} \mathrm{kg}^{-1}\right)\end{array}$ & $\begin{array}{c}\mathrm{T} \\
\left(\mathrm{cmol}_{\mathrm{c}} \mathrm{kg}^{-1}\right)\end{array}$ & $\begin{array}{l}\mathrm{Fe}_{2} \mathrm{O}_{3} \\
\left(\mathrm{~g} \mathrm{~kg}^{-1}\right)\end{array}$ \\
\hline \multicolumn{11}{|c|}{ Área I, Argissolo Amarelo distrocoeso típico, pedoforma côncava } \\
\hline$A+E$ & $0-82$ & 10YR 5/1 & 693 & 122 & 185 & 1,52 & 4,9 & 12,3 & 3,5 & 5,2 \\
\hline Bt2 & $82-140$ & 10YR $8 / 3$ & 546 & 155 & 299 & 1,56 & 5,5 & 4,0 & 1,9 & 8,8 \\
\hline \multicolumn{11}{|c|}{ Área II, Argissolo Amarelo distrocoeso típico, pedoforma côncava } \\
\hline $\mathrm{A}+\mathrm{E}$ & $0-85$ & 10YR 4/1 & 714 & 107 & 179 & 1,54 & 5,2 & 9,7 & 3,5 & 6,1 \\
\hline Bt2 & $85-130$ & $10 \mathrm{YR} 8 / 4$ & 574 & 128 & 298 & 1,52 & 5,3 & 4,0 & 1,7 & 12,3 \\
\hline \multicolumn{11}{|c|}{ Área III, Argissolo Amarelo distrocoeso típico, pedoforma convexa } \\
\hline$A+E$ & $0-60$ & 7,5 YR $7 / 3$ & 617 & 170 & 213 & 1,52 & 5,4 & 9,5 & 2,2 & 10,1 \\
\hline Bt2 & $100+$ & 7,5 YR $8 / 6$ & 497 & 165 & 338 & 1,40 & 4,7 & 2,0 & 1,8 & 17,7 \\
\hline
\end{tabular}

${ }^{(1)}$ Hor., horizontes do solo; Prof., profundidade; Cor, cor determinada pela carta de cores de Munsell, por amostra seca; DS, densidade do solo; $\mathrm{pH}$, $\mathrm{pH}$ em $\mathrm{H}_{2} \mathrm{O}$; MO, matéria orgânica, cujo valor foi obtido pela multiplicação do teor de carbono orgânico total por 1,724; $\mathrm{T}$, capacidade de troca catiônica; $\mathrm{Fe}_{2} \mathrm{O}_{3}$, teor de ferro pelo ataque sulfúrico. Tabela adaptada de Dantas et al. (2014). 
( $\mathrm{kg} \quad \mathrm{s} \mathrm{m}^{-4}$ ) expressa o potencial do solo em ser desagregado pela chuva e transportado pelo escoar de uma delgada lâmina de água. O coeficiente de ES (s $\mathrm{m}^{-1}$ ) expressa o potencial do solo em ser desagregado em pequenos canais que se formam.

Para a obtenção dos espectros de reflectância difusa, as avaliações foram feitas com o sensor de laboratório Lambda 950 (PerkinElmer do Brasil, São Paulo, SP). Foi moído aproximadamente $0,5 \mathrm{~g}$ de TFSA em cadinho de ágata até a obtenção de coloração constante. O conteúdo foi colocado em porta-amostras com espaço cilíndrico de $16 \mathrm{~mm}$. Os valores de reflectância foram determinados em espectrofotômetro equipado com esfera integradora de $80 \mathrm{~mm}$, a cada $1 \mathrm{~nm}$, com tempo de integração de $0,2 \mathrm{~s}$, com varredura no intervalo de 380 a $780 \mathrm{~nm}$. Após a obtenção dos espectros, foram determinados os valores de triestímulo XYZ, definidos pela Comisión Internacional de L'Eclairage-CIE (Wyszecki \& Stiles, 1982). A partir das coordenadas $\mathrm{XYZ}$, foram deduzidos os valores Munsell de matiz, croma e valor, com auxílio do programa Munsell Conversion, versão 6.4, conforme Barrón et al. (2000). Com base nos valores de matiz, valor e croma obtidos pela análise de espectroscopia de refletância difusa, foi calculado o índice de avermelhamento (IAV), de acordo com Barrón et al. (2000): IAV $=((10-\mathrm{H}) / \mathrm{V}) \times \mathrm{C}$, em que $\mathrm{V}$ e $\mathrm{C}$ são os valores numéricos do valor e do croma Munsell, respectivamente, e $\mathrm{H}$ corresponde ao número que procede o YR no matiz.

Os dados foram avaliados pela análise de variância e pelo teste $\mathrm{F}$, ao se considerar as diferentes áreas estudadas como fator de variação. A comparação entre as médias foi realizada por meio do teste de Tukey, a $1 \%$ de probabilidade.

Após estimar os valores dos coeficientes de EE e ES por meio das equações propostas por Flanagan \& Livingston (1995), foram modeladas funções de pedotransferência para $90 \%$ do total de pontos coletados $(n=327)$, tendo-se utilizado os valores de EE e ES como variáveis dependentes e os parâmetros da cor como variáveis independentes (Tabela 2).

Os modelos com maiores valores de $\mathrm{R}^{2}$ foram utilizados para estimar os valores dos coeficientes de EE e ES para o restante dos $10 \%$ dos pontos coletados $(n=36)$. Esse procedimento foi realizado para evitar erros de retroalimentação na validação do modelo ajustado (Siqueira et al., 2010). Os valores de EE e ES estimados para os 36 pontos com uso dos parâmetros de valor e croma foram comparados aos valores de EE e ES calculados pelas equações de Flanagan \& Livingston (1995), ao se utilizar areia muito fina e matéria orgânica.

Na validação externa, os coeficientes de determinação $\left(\mathrm{R}^{2}\right)$ e a raiz do erro quadrático médio (RMSE) foram utilizados para avaliar, respectivamente, a precisão e a acurácia entre os valores de EE e ES estimados com base em areia muito fina e matéria orgânica, e os estimados com base em valor e croma:

$$
\text { RMSE }=\frac{\left\{\frac{1}{n} \sum_{i=1}^{n}\left[y\left(x_{i}\right)-\hat{y}\left(x_{i}\right)\right]^{2}\right\}^{0,5}}{\sigma_{i}}
$$

em que $\mathrm{n}$ é o número de amostras; $\mathrm{y}\left(\mathrm{x}_{\mathrm{i}}\right)$ é o valor observado da propriedade no ponto $\mathrm{i} ; \hat{\mathrm{y}}(\mathrm{xi})$ é o valor estimado da propriedade no ponto $i$; e $\sigma$ é o desvio-padrão das variáveis observadas.

\section{Resultados e Discussão}

Os maiores valores médios de EE e ES foram obtidos na área III (Tabela 3). Esses altos valores podem ser atribuídos ao fato de esta área estar localizada em pedoforma convexa, o que propicia maiores perdas de solo (Daniels et al., 1987). A pedoforma côncava apresenta maior remoção nas partes mais altas e maior acúmulo nas áreas mais baixas (Resende et al., 2007). Sanchez et al. (2009), ao estudarem a variabilidade espacial dos fatores de erosão em diferentes pedoformas, encontraram maior perda de solo na pedoforma convexa $\left(9,32 \mathrm{Mg} \mathrm{ha}^{-1}\right.$ por ano) do que na côncava (7,21 $\mathrm{Mg} \mathrm{ha}^{-1}$ por ano), em Argissolos.

Tabela 2. Modelos para a estimativa da erodibilidade em entressulcos (EE) e sulcos (ES) de Argissolos coesos do Leste Maranhense ${ }^{(1)}$.

\begin{tabular}{ccc}
\hline Modelo & Função de pedotransferência & $\mathrm{R}^{2}$ \\
\hline 1 & $\mathrm{EE}=1442923+828662$ valor & $0,70^{*}$ \\
2 & $\mathrm{EE}=3529641+782088 \mathrm{croma}$ & $0,64^{*}$ \\
3 & $\mathrm{EE}=16178411-1121418 \mathrm{matiz}$ & $0,44^{*}$ \\
4 & $\mathrm{EE}=5079166+1670378 \mathrm{IAV}$ & $0,49^{*}$ \\
5 & $\mathrm{ES}=-0,0118+0,00279$ valor & $0,69^{*}$ \\
6 & $\mathrm{ES}=-0,00537+0,00283 \mathrm{croma}$ & $0,72^{*}$ \\
7 & $\mathrm{ES}=0,0352-0,00349$ matiz & $0,37^{*}$ \\
8 & $\mathrm{ES}=0,000513+0,00551 \mathrm{IAV}$ & $0,46^{*}$ \\
\hline
\end{tabular}

(1)IAV, índice de avermelhamento. *Significativo pelo teste F, a $5 \%$ de probabilidade. $\mathrm{n}=327$. 
Os componentes da cor do solo (matiz, valor e croma) determinados pela espectroscopia de reflectância difusa apresentaram diferenças entre as áreas estudadas. Os solos das áreas I e II apresentaram coloração mais amarelada, enquanto a área III apresentou coloração mais avermelhada; o índice de avermelhamento foi de 0,89, 0,33 e 0,24 nas áreas III, I e II, respectivamente.

O valor, o croma e o índice de avermelhamento apresentaram diferenças significativas entre as áreas, e os maiores valores médios foram obtidos na área III. Os solos das áreas I e II estão situados em pedoformas côncavas, e o solo da área III se situa em pedoforma convexa. A pedoforma côncava favorece o armazenamento de água e, consequentemente, reações de redução (Resende et al., 2007). Nesse ambiente, o $\mathrm{Fe}^{+++}$, responsável pela cor vermelha do solo, é reduzido a $\mathrm{Fe}^{++}$, o qual é mais solúvel e, portanto, facilmente retirado do sistema, o que faz com que o solo permaneça com coloração mais amarelada (Brady \& Weil, 2013).

Em solos sujeitos aos ciclos de umedecimento e secagem, há redução e oxidação do íon ferro, com sua posterior argiluviação. Nesses ambientes, ocorre o processo de erosão seletiva, que se manifesta por meio do carreamento preferencial de partículas finas, em razão do fluxo superficial de água e, também, das partículas grosseiras. Já nos horizontes subsuperficiais, o processo de neoformação de argila pode ocorrer em determinadas condições. Em ambientes de redução, a dissolução e o transporte de $\mathrm{Fe}^{++}$, bem como de outros cátions, para horizontes mais profundos, constituem processo preferencial de enriquecimento de materiais em subsuperfície (Tabela 1) (Brady \& Weil, 2013).

Tabela 3. Valores médios da erodibilidade em entressulcos (EE) e sulcos (ES), e parâmetros da cor (matiz, valor e croma) obtidos por espectroscopia de reflectância difusa e índice de avermelhamento (IAV), em Argissolos coesos do Leste Maranhense ${ }^{(1)}$.

\begin{tabular}{lcccccc}
\hline Área & $\mathrm{EE}\left(\mathrm{kg} \mathrm{s} \mathrm{m}^{-4}\right)$ & $\mathrm{ES}\left(\mathrm{s} \mathrm{m}^{-1}\right)$ & Matiz & Valor & Croma & IAV \\
\hline I & $5696187 \mathrm{~b}$ & $1,23 \times 10^{-3} \mathrm{c}$ & $9,33 \mathrm{~b}$ & $4,82 \mathrm{~b}$ & $2,34 \mathrm{c}$ & $0,33 \mathrm{~b}$ \\
II & $5139365 \mathrm{c}$ & $2,20 \times 10^{-3} \mathrm{~b}$ & $9,55 \mathrm{a}$ & $4,90 \mathrm{~b}$ & $2,62 \mathrm{~b}$ & $0,24 \mathrm{c}$ \\
III & $6843010 \mathrm{a}$ & $6,15 \times 10^{-3} \mathrm{a}$ & $8,62 \mathrm{c}$ & $6,42 \mathrm{a}$ & $4,12 \mathrm{a}$ & $0,89 \mathrm{a}$ \\
\hline $\mathrm{F}^{(2)}$ & $610,27^{* *}$ & $270,89^{* *}$ & $354,41^{* *}$ & $876,13^{* *}$ & $2.036,31^{* *}$ & $434,16^{* *}$ \\
$\mathrm{CV}(\%)$ & 6,37 & 52,51 & 3,00 & 6,11 & 7,53 & 36,46 \\
\hline
\end{tabular}

(1)Médias seguidas de letras iguais não diferem pelo teste de Tukey, a $1 \%$ de probabilidade. ${ }^{(2)} \mathrm{F}$, valores do teste $\mathrm{F}$ da análise de variância. ${ }^{* *}$ Significativo a $1 \%$ de probabilidade. $n=121$.
Todos os atributos avaliados produziram modelos lineares simples significativos, o que evidenciou a dependência dos coeficientes de EE e ES com a cor do solo (Tabela 2). Os atributos valor e croma destacaramse como as variáveis preditoras que proporcionaram maiores valores de $\mathrm{R}^{2}(0,70$ e 0,72$)$ na predição de $\mathrm{EE}$ e ES, respectivamente. De acordo com a classificação de Warrick \& Nielsen (1980), os atributos EE, matiz, valor e croma apresentam baixa variabilidade $(\mathrm{CV} \leq 12 \%)$, enquanto a ES e o índice de avermelhamento se enquadram na classe de alta variabilidade $(\mathrm{CV} \geq 24 \%)$. Os altos valores de CV encontrados para ES são característicos dessa propriedade (Singh et al., 2012).

A presença do croma no modelo final para a estimativa do coeficiente de ES pode estar relacionada aos baixos teores de MO (Tabela 1). Demattê et al. (2011) verificaram que os valores dos componentes valor $(\mathrm{r}=0,60)$ e croma $(\mathrm{r}=0,79)$ são inversamente proporcionais ao teor de MO do solo. Esses autores também constataram que o componente valor, isoladamente, não foi adequado para a predição da MO no solo; no entanto, em conjunto com o croma, completou o modelo de predição $\left(\mathrm{R}^{2}=0,66\right)$. Esse padrão pode ser confirmado visualmente na carta de cores de Munsell, cujos valores mais elevados de valor e croma, em solos de cores mais claras, geralmente correspondem a menores teores de MO.

Ao se comparar as duas formas de predição utilizadas no presente trabalho, foram obtidos valores de $\mathrm{R}^{2}$ de 0,70 e 0,73 , e de RMSE de 54 e $53 \%$ para EE e ES, respectivamente (Figura 2). Menores valores de RMSE estão associados a modelos mais acurados de predição; valores próximos a $40 \%$ indicam precisão satisfatória, com valores de $\mathrm{R}^{2}$ próximos de $85 \%$, enquanto valores de RMSE $>71 \%$ indicam que o modelo representa menos de $50 \%$ da variabilidade dos pontos de validação (Hengl, 2009). Assim, os valores observados no presente trabalho mostram que os modelos gerados apresentaram acurácia e precisão na predição dos valores de EE e ES, a partir das funções de pedotransferência obtidas.

Os resultados encontrados são indicativos de que o uso dos componentes da cor, determinados pela espectroscopia de reflectância difusa, é alternativa promissora nos estudos da erodibilidade em sulcos e entressulcos, além de ser boa preditora para a estimativa desses atributos no Leste Maranhense. A alta sensibilidade dessa metodologia pode auxiliar 
na identificação e no mapeamento de ambientes com características específicas da região tropical, principalmente de regiões que apresentam baixos teores de ferro, como Argissolos e Latossolos originados de sedimentos da Formação Barreiras.

A determinação da cor do solo por espectroscopia de reflectância difusa, aliada à identificação das pedoformas, também pode ser utilizada para identificar áreas propícias à erodibilidade, as quais devem apresentar manejos específicos, com vistas à redução das perdas de solo pela erosão.

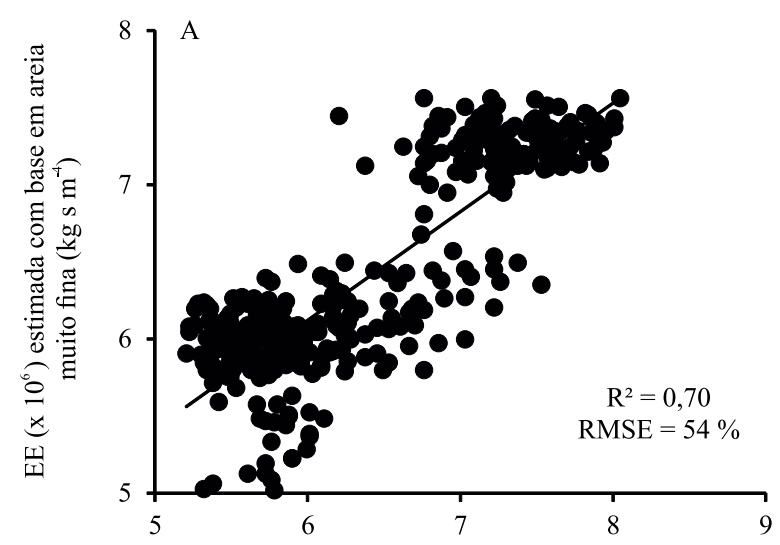

EE $\left(\times 10^{6}\right)$ estimada com base em parâmetro de valor do modelo $1\left(\mathrm{~kg} \mathrm{~s} \mathrm{~m}^{-4}\right)$

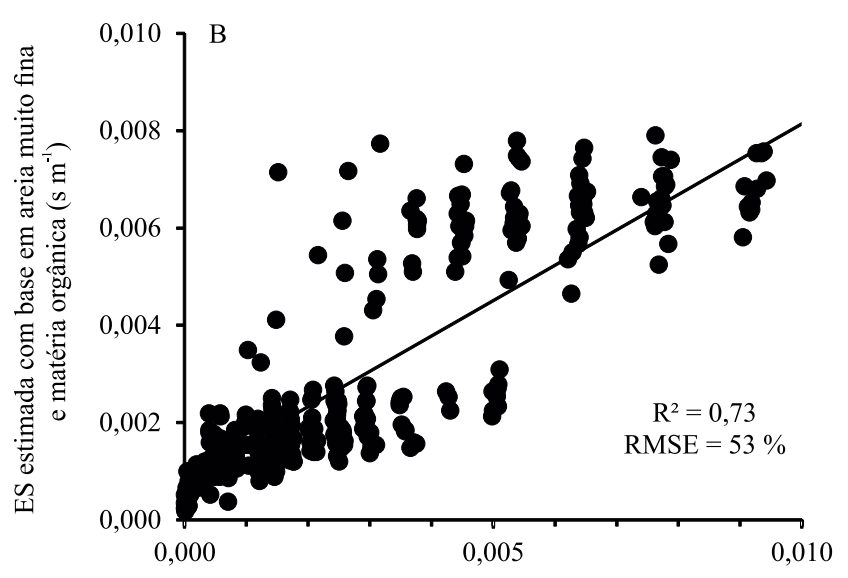

ES estimada com base em parâmetro de croma do modelo $6\left(\mathrm{~s} \mathrm{~m}^{-1}\right)$

Figura 2. Relação entre os valores dos coeficientes de erodibilidade em entressulcos (EE) (A) e sulcos (ES) (B) estimados pela equação de Flanagan \& Livingston (1995) e os estimados pelas funções de pedotransferência (modelos 1 e 6 da Tabela 2), para Argissolos coesos do Leste Maranhense.

\section{Conclusão}

Os parâmetros valor e croma da cor do solo, quantificados pela espectroscopia de reflectância difusa, podem ser utilizados para predizer a erodibilidade em sulcos e entressulcos de Argissolos coesos do Leste Maranhense.

\section{Agradecimentos}

À Coordenação de Aperfeiçoamento de Pessoal de Nível Superior (Capes) e ao Conselho Nacional de Desenvolvimento Científico e Tecnológico (CNPq), pela concessão de bolsas.

\section{Referências}

ANDRADE, N.S.F. de; MARTINS FILHO, M.V.; TORRES, J.L.R.; PEREIRA, G.T.; MARQUES JÚNIOR, J. Impacto técnico e econômico das perdas de solo e nutrientes por erosão no cultivo da cana-de-açúcar. Engenharia Agrícola, v.31, p.539-550, 2011. DOI: $10.1590 /$ S0100-69162011000300014.

ATLAS do Maranhão. 2.ed. São Luís: Gerência de Planejamento e Desenvolvimento Econômico, 2002. 39p.

BARRÓN, V.; MELLO, J.W.V.; TORRENT, J. Caracterização de óxidos de ferro em solos por espectroscopia de reflectância difusa. In: NOVAIS, R.F. de; ALVAREZ V., V.H.; SCHAEFER, C.E.G.R. (Ed.). Tópicos em ciência do solo. Viçosa: Sociedade Brasileira de Ciência do Solo, 2000. p.139-162.

BRADY, N.C.; WEIL, R.R. Elementos da natureza e propriedades dos solos. 3.ed. Porto Alegre: Bookman, 2013. 704p.

CASSOL, E.A.; LIMA, V.S. de. Erosão em entressulcos sob diferentes tipos de preparo e manejo do solo. Pesquisa Agropecuária Brasileira, v.38, p.117-124, 2003. DOI: 10.1590/ S0100-204X2003000100016.

COHEN, J.; BARLOW, M.; KUSHNER, P.J.; SAITO, K. Stratosphere-troposphere coupling and links with Eurasian land surface variability. Journal of Climate, v.20, p.5335-5343, 2007. DOI: 10.1175/2007JCLI1725.1.

DANIELS, R.B.; GILLIAN, J.W.; CASSEL, D.K.; NELSON, L.A. Quantifying the effects of past soil erosion on present soil productivity. Journal of Soil and Water Conservation, v.42, p.183-187, 1987.

DANTAS, J.S.; MARQUES JÚNIOR, J.; MARTINS FILHO, M.V.; RESENDE, J.M. do A.; CAMARGO, L.A.; BARBOSA, R.S. Gênese de solos coesos do Leste Maranhense: relação solo-paisagem. Revista Brasileira de Ciência do Solo, v.38, p.1039-1050, 2014. DOI: 10.1590/S010006832014000400001 .

DEMATTÊ, J.A.M.; BORTOLETTO, M.A.M.; VASQUES, G.M.; RIZZO, R. Quantificação de matéria orgânica do solo através de modelos matemáticos utilizando colorimetria no sistema Munsell 
de cores. Bragantia, v.70, p.590-597, 2011. DOI: 10.1590/ S0006-87052011005000006.

DONAGEMA, G.K.; CAMPOS, D.V.B. de; CALDERANO, S.B.; TEIXEIRA, W.G.; VIANA, J.H.M. (Org.). Manual de métodos de análise de solos. 2.ed. Rio de Janeiro: Embrapa Solos, 2011. 230p. (Embrapa Solos. Documentos, 132).

FLANAGAN, D.C.; LIVINGSTON, S.J. USDA - water erosion prediction project: WEPP user summary. West Lafayette: USDA-ARS National Soil Research Laboratory, 1995. 141p. (NSERL. Report, 11).

HENGL, T. A practical guide to geoestatistical mapping. 2.ed. Amsterdam: University of Amsterdam, 2009. 291p.

JACOMINE, P.K.T.; CAVALCANTI, A.C.; PESSÔA, S.C.P.; BURGOS, N.; MEDEIROS, L.A.R.; LOPES, O.F.; MELO FILHO, H.F.R. Levantamento exploratório-reconhecimento de solos do Estado do Maranhão. Rio de Janeiro: Embrapa-SNLCS: Sudene-DRN, 1986. 964p. (Embrapa SNLCS. Boletim de pesquisa, 35). (Sudene-DRN. Série recursos de solos, 17).

LANE, L.J.; NEARING, M.A. USDA - water erosion prediction project: hillslope profile model documentation. West Lafayette: USDA-ARS National Soil Research Laboratory, 1989. 233p. (NSERL report, 2).

MARTINS FILHO, M.V.; LICCIOTI, T.T.; PEREIRA, G.T.; MARQUES JUNIOR, J.; SANCHEZ, R.B. Perdas de solo e nutrientes por erosão num Argissolo com resíduos vegetais de cana-de-açúcar. Engenharia Agrícola, v.29, p.8-18, 2009. DOI: 10.1590/S0100-69162009000100002.

MCBRATNEY, A.B.; MINASNY, B.; CATTLE, S.R.; VERVOORT, R.W. From pedotransfer functions to soil inference systems. Geoderma, v.109, p.41-73, 2002. DOI: 10.1016/ S0016-7061(02)00139-8.

MINASNY, B.; HARTEMINK, A.E. Predicting soil properties in the tropics. Earth-Science Reviews, v.106, p.52-62, 2011. DOI: 10.1016/j.earscirev.2011.01.005.

PARSONS, A.J.; WAINWRIGHT, J.; FUKUWARA, T.; ONDA, Y. Using sediment travel distance to estimate medium-term erosion rates: a 16-year record. Earth Surface Processes and Landforms, v.35, p.1694-1700, 2010. DOI: 10.1002/esp.2011.

PELUCO, R.G.; MARQUES JUNIOR, J.; SIQUEIRA, D.S.; PEREIRA, G.T.; BARBOSA, R.S.; TEIXEIRA, D. de B.; ADAME, C.R.; CORTEZ, L.A. Suscetibilidade magnética do solo e estimação da capacidade de suporte à aplicação de vinhaça. Pesquisa Agropecuária Brasileira, v.48, p.661-672, 2013. DOI: 10.1590/S0100-204X2013000600012.

QUINTON, J.N.; GOVERS, G.; VAN OOST, K.; BARDGETT, R.D. The impact of agricultural soil erosion on biogeochemical cycling. Nature Geoscience, v.3, p.311-314, 2010. DOI: 10.1038/ngeo838.

RAIJ, B. van; ANDRADE, J.C. de; CANTARELA, H.; QUAGGIO, J.A. (Ed.). Análise química para avaliação da fertilidade de solos tropicais. Campinas: Instituto Agronômico, 2001. 285p.

RESENDE, M.; CURI, N.; REZENDE, S.B. de; CORREAA, G.F. Pedologia: base para distinção de ambientes. 5.ed. Lavras: Ed. da UFV, 2007. 322p.
ROCKSTROM, J.A.; STEFFEN, W.; NOONE, K.; PERSSON, Å.; CHAPIN, F.S.; LAMBIN, E.F.; LENTON, T.M.; SCHEFFER, M.; FOLKE, C.; SCHELLNHUBER, H.J.; NYKVIST, B.; WIT, C.A. de; HUGHES, T.; LEEUW, S. van der; RODHE, H.; SÖRLIN, S.; SNYDER, P.K.; COSTANZA, R.; SVEDIN, U.; FALKENMARK, M.; KARLBERG, L.; CORELL, R.W.; FABRY, V.J.; HANSEN, J.; WALKER, B.; LIVERMAN, D.; RICHARDSON, K.; CRUTZEN, P.; FOLEY, J.A. A safe operating space for humanity. Nature, v.461, p.472-475, 2009. DOI: $10.1038 / 461472 \mathrm{a}$.

SANCHEZ, R.B.; MARQUES JÚNIOR, J.; PEREIRA, G.T.; DE SOUZA, Z.M.; PEREIRA, G.T.; MARTINS FILHO, M.V. Variabilidade espacial de atributos do solo e de fatores de erosão em diferentes pedoformas. Bragantia, v.68, p.1095-1103, 2009. DOI: $10.1590 / \mathrm{S} 0006-87052009000400030$.

SANTOS, G.G.; GRIEBELER, N.P.; OLIVEIRA, L.F.C. de. Chuvas intensas relacionadas à erosão hídrica. Revista Brasileira de Engenharia Agrícola e Ambiental, v.14, p.115-123, 2010. DOI: $10.1590 /$ S1415-43662010000200001.

SANTOS, H.G. dos; JACOMINE, P.K.T.; ANJOS, L.H.C. dos; OLIVEIRA, V.A. de; OLIVEIRA, J.B. de; COELHO, M.R.; LUMBRERAS, J.F.; CUNHA, T.J.F. (Ed.). Sistema brasileiro de classificação de solos. 2.ed. Rio de Janeiro: Embrapa Solos, 2006. 306p.

SANTOS, H.L.; MARQUES JÚNIOR, J.; MATIAS, S.S.R.; SIQUEIRA, D.S.; MARTINS FILHO, M.V. Erosion factors and magnetic susceptibility in differet compartments of a slope in Gilbués-PI, Brazil. Engenharia Agrícola, v.33, p.64-74, 2013. DOI: $10.1590 / \mathrm{S} 0100-69162013000100008$.

SHERIDAN, G.J.; SO, H.B.; LOCH, R.J.; WALKER, C.M. Estimation of erosion model erodibility parameters from media properties. Australian Journal of Soil Research, v.38, p.129-136, 2000. DOI: 10.1071/SR99041.

SINGH, R.K.; PANDA, R.K.; SATAPATHY, K.K.; NGACHAN, S.V. Runoff and sediment yield modelling for a treated hilly watershed in Eastern Himalaya using the water erosion prediction project model. Water Resources Management, v.26, p.643-665, 2012. DOI: $10.1007 / \mathrm{s} 11269-011-9937-4$.

SIQUEIRA, D.S.; MARQUES JÚNIOR, J.; PEREIRA, G.T. The use of landforms to predict the variability of soil and orange attributes. Geoderma, v.155, p.55-66, 2010. DOI: 10.1016/j. geoderma.2009.11.024.

TORRENT, J.; BARRÓN, V. Diffuse reflectance spectroscopy. In: ULERY, A.L.; DREES, L.R. (Ed.). Methods of soil analysis. Part 5 - mineralogical methods. Madison: Soil Science Society of America, 2008. p.367-387.

VISCARRA ROSSEL, R.A.; WEBSTER, R. Discrimination of Australian soil horizons and classes from their visible-near infrared spectra. European Journal of Soil Science, v.62, p.637-647, 2011. DOI: 10.1111/j.1365-2389.2011.01356.x.

WARRICK, A.W.; NIELSEN, D.R. Spatial variability of soil physical properties in the field. In: HILLEL, D. (Ed.). Applications of soil physics. New York: Academic, 1980. p.319-344. DOI: 10.1016/B978-0-12-348580-9.50018-3. 
WYSZECKI, G.; STILES, W.S. Color science: concepts and methods, quantitative data and formulae. $2^{\text {nd }}$ ed. New York: J. Wiley, 1982.968p.
WISCHMEIER, W.H.; SMITH, D.D. Predicting rainfall erosion losses: a guide to conservation planning. Washington: U.S. Department of Agriculture, 1978. 58p.

$\overline{\text { Recebido em } 19 \text { de dezembro de } 2013 \text { e aprovado em } 5 \text { de setembro de } 2014}$ 